\title{
Trend of HPV 16/18 Genotypes in Cervical Intraepithelial Neoplasia Grade 3: Data for 2007-2018
}

\author{
Luca Giannella' \\ Giovanni Delli Carpini' \\ Jacopo Di Giuseppe' \\ Giorgio Bogani $\mathbb{D}^{2}$ \\ Barbara Gardella ${ }^{3}$ \\ Ermelinda Monti ${ }^{4}$ \\ Carlo Antonio Liverani (iD ${ }^{4}$ \\ Alessandro Ghelardi ${ }^{5}$ \\ Salvatore Insinga' \\ Michele Montanari' \\ Francesco Raspagliesi ${ }^{2}$ \\ Arsenio Spinillo ${ }^{3}$ \\ Paolo Vercellini ${ }^{4}$ \\ Elena Roncella ${ }^{4}$ \\ Andrea Ciavattini ${ }^{1}$ \\ 'Woman's Health Sciences Department, \\ Gynecologic Section, Polytechnic \\ University of Marche, Ancona, Italy; \\ ${ }^{2}$ Gynecologic Oncology Unit, Fondazione \\ IRCCS Istituto Nazionale dei Tumori di \\ Milano, Milano, Italy; ${ }^{3}$ Department of \\ Obstetrics and Gynecology, Fondazione \\ IRCCS Policlinico San Matteo, Università \\ degli Studi di Pavia, Pavia, Italy; \\ ${ }^{4}$ Gynaecology Unit, Fondazione IRCCS \\ Ca' Granda Ospedale Maggiore \\ Policlinico, Milano, Italy; ${ }^{5}$ Azienda UsI \\ Toscana Nord-Ovest, UOC Ostetricia \\ e Ginecologia, Ospedale Apuane, Massa, \\ Italy
}

Correspondence: Andrea Ciavattini Woman's Health Sciences Department, Gynecologic Section, Polytechnic University of Marche, via Filippo Corridoni, 16, Ancona, 60123, Italy

Tel +3907I5962I72

Fax +3907/36576

Email ciavattini.a@libero.it
Aim: In the post-vaccination era, the starting age and time intervals of cervical screening could change (older age and longer screening intervals). This scenario may be achieved by significantly reducing human papillomavirus (HPV) 16/18 prevalence (genotypes included in the current vaccines). In this regard, assessing the trend over time of these HPV infections in high-grade cervical lesions can provide information on the objective. The present study aimed to evaluate the trend of HPV 16/18 over the years 2007-2018 in women with cervical intraepithelial neoplasia (CIN) grade 3.

Methods: This is a retrospective multi-institutional study including HPV genotyped and unvaccinated women under 30 with CIN3. The sample was divided into the following periods: 2007-2010, 2011-2014, 2015-2018. HPV genotypes were grouped in genotypes $16 / 18$, genotypes $31 / 33 / 35 / 52 / 58 / 67$ (genetically related to HPV16), genotypes 39/45/59/68/ 70 (genetically related to HPV18), genotypes 31/33/45/52/58 (high-risk types included in the nonavalent vaccine), possibly carcinogenic HPV (genotypes 26/30/53/67/70/73/82/85), lowrisk HPV (genotypes 6/11/40/42/43/44/54/55/61). The trend between periods and HPV genotypes was measured using the Cochran-Armitage test for trend.

Results: The final analysis included 474 participants. HPV 16/18 prevalence decreased significantly over the years $(77.8 \%$ vs $68.9 \%$ vs $66.0 \%$, respectively, Ptrend $=0.027)$. Possibly carcinogenic HPV (genotypes 26/30/53/67/70/73/82/85) showed a significant negative prevalence trend over time $(4.9 \%$ vs $1.1 \%$ vs $1.3 \%$, respectively, Ptrend=0.046). Finally, there was a significant positive trend over the years for highrisk HPV genotypes 31/33/45/52/58 in women under $25(9.9 \%$ vs $17.0 \%$ vs $24.0 \%$, respectively, Ptrend=0.048).

Conclusion: The prevalence of CIN3 lesions related to HPV 16/18 genotypes decreased over time from 2007 to 2018. These data highlight a herd effect of the HPV vaccine. However, fifteen years after HPV vaccine introduction, we are still a long way from herd immunity. The increase in high-risk types 31/33/45/52/58 will need to be reassessed when the nonavalent vaccine impact will be more reliable.

Keywords: cervical cancer, cervical intraepithelial neoplasia, cervical cancer screening program, HPV vaccine, HPV-16/18

\section{Introduction}

Cervical cancer (CC) is one of the most common female cancers in developing countries. ${ }^{1}$ Human papillomavirus (HPV) infection is a necessary factor for cellular neoplastic transformation. ${ }^{2}$ The HPV vaccination and screening programs represent primary and secondary prevention measures. ${ }^{2}$ 
There are three HPV vaccines with an established benefit-risk ratio. ${ }^{3-8}$ In 2006-2007 the Food and Drug Administration (FDA) approved the bivalent and quadrivalent HPV vaccines (including HPV 16/18 and HPV 6/ 11/16/18, respectively). ${ }^{4}$ Afterward, the FDA approved the nonavalent vaccine in 2014 (including HPV 6/11/16/18/ $31 / 33 / 45 / 52 / 58){ }^{7}$ For the latter, it will be necessary to wait for the next few years to assess its true impact.

Conversely, several studies evaluated the effect of the bivalent and quadrivalent vaccines. It was reported significant reductions in the prevalence of vaccine-related genotypes in both vaccinated and unvaccinated women (herd protection) after the HPV vaccine introduction. ${ }^{9}$ Vaccinated and unvaccinated women showed an $80 \%$ and $40 \%$ reduction in HPV genotypes of the 4-valent vaccine, respectively. ${ }^{9}$

There is no definitive evidence for HPV type replacement following the introduction of HPV vaccines. Some authors showed a competitive advantage of non-vaccine HPV types, while other studies reported not-types replacement after HPV vaccination introduction. ${ }^{10-12}$ It is crucial to monitor all these trends to optimize vaccination and cervical cancer screening policies.

Estimates indicate a significant and continuous reduction in mortality and incidence of CC in Italy. Based on data available up to 2012, the drop was more pronounced between 1980 and 2000 and continues, albeit to a lesser extent, until 2012. The standardized incidence rate was 14 per 100.000 women in 1980 and 6 or 4 per 100.000 in 2002 and 2012, respectively. ${ }^{13}$ In Italy, the HPV vaccination was introduced in 2007/2008, and the age range for administration is $9-14$ years. ${ }^{4}$ Since 2017 , the nonavalent vaccine has become available. ${ }^{4}$ As of 31 December 2018, anti-HPV vaccination coverage in the female population (birth cohorts 1997-2006) amounted to 61.7\% for the first dose and $40.3 \%$ for the complete cycle. ${ }^{14}$ In Europe, vaccination coverage has an average of $37 \%$, ranging between $30-43 \% .^{2}$ In North America, the mean is 54\%, ranging between $27-86 \%{ }^{2}$ The objective to be achieved should be a minimum vaccination coverage threshold around $95 \%{ }^{15}$

To date, vaccine status does not affect CC screening programs. ${ }^{16}$ They include HPV testing/genotyping and cytology to determine the risk of cervical intraepithelial neoplasia (CIN) grade 3 as the best surrogate of CC risk. Currently, CC screening begins at 25 years with time intervals of 3-5 years, according to cytology or HPV test use, respectively. ${ }^{16}$ Only when vaccination impact will significantly reduce HPV 16/18 infections (genotypes included in currently used vaccines), vaccinated and unvaccinated women can be screened equally at an older age and with longer screening intervals. In this regard, assessing HPV16/18 prevalence over time in women with high-grade cervical lesions can provide information on the objective.

The present study mainly aimed to assess the trend of HPV 16/18 over the years 2007-2018 in a real-world setting of women under 30 years with CIN3. We also evaluated the trend of the following HPVs: Genotypes 31/33/35/52/58/67 (genetically related to HPV16), genotypes 39/45/59/68/70 (genetically related to HPV18), genotypes 31/33/45/52/58 (high-risk-types included in the nonavalent vaccine), possibly carcinogenic HPV (genotypes 26/30/53/67/70/73/82/85), low-risk HPV (genotypes $6 / 11 / 40 / 42 / 43 / 44 / 54 / 55 / 61$ ). Finally, the trend of multiple HPV infections was also measured.

\section{Materials and Methods Study Design and Setting}

This retrospective multi-institutional study included unvaccinated women undergoing loop electrosurgical excision procedures between March 2007 and March 2018. Inclusion criteria:

- Women with a CIN3 on cone histology;

- Age under 30;

- Available HPV genotyping before surgery.

Participants aged 30 years and over with previous cervical excisional treatment, immunological disease, pregnancy were excluded.

Based on Italian law, the Ethics Committee (Comitato Etico Regionale Marche) took note of the study (Prot. 10/ 2021). ${ }^{17}$ According to Italian law, patient consent was not mandatory. ${ }^{17}$

The Centers participating in the study manage women included in both opportunistic and organized cervical cancer screening programs. HPV genotyping is usually performed in these centers as a management aid: 1) every 12 months during follow-up; 2) before surgical treatment (conization).

\section{Variables}

The study period was divided into three groups: $2007-$ 2010, 2011-2014, 2015-2018. The age-related impact of 
HPV vaccination over time was assessed. According to age, the participants were categorized as women $<25$ and 25-29. Finally, the same trends were analyzed for multiple HPV infections.

According to HPV genotype classifications, ${ }^{18,19}$ they were classified as follows: 1) high-risk HPV (genotypes $16 / 18 / 31 / 33 / 35 / 39 / 45 / 51 / 52 / 56 / 58 / 59 / 66 / 68)$; 2) possibly carcinogenic HPVs (genotypes 26/30/53/67/70/73/82/ 85); 3) low-risk HPV (genotypes 6/11/40/42/43/44/54/55/ 61 ). Previous studies ${ }^{20,21}$ have used hierarchical attribution in cases of multiple HPV infections. Based on this criterion, the CIN3 lesion was attributed to the genotype most associated with precancerous lesions of the uterine cervix: HPV 16/18 > all other HPVs; high-risk HPV > possibly carcinogenic HPV; possibly carcinogenic HPV > low-risk HPV. HPV genotypes were grouped in genotypes $16 / 18$, genotypes 31/33/35/52/58/67 (genetically related to HPV16), genotypes 39/45/59/68/70 (genetically related to HPV18), genotypes 31/33/45/52/58 (high-risk-types included in the nonavalent vaccine), possibly carcinogenic HPV (genotypes 26/30/53/67/70/73/82/85), low-risk HPV (genotypes 6/11/40/42/43/44/54/55/61). The prevalence of each high-risk HPV genotype was reported considering its presence in both single and multiple infections.

\section{Data Source/Measurements}

The data of interest were recovered from the databases of each Center participating in the study and made anonymous regarding the patients' privacy. Cytological sampling was performed using an endocervical swab and Thin Prep (Hologic, Marlborough, MA, USA). Per local protocols, DNA extraction and HPV genotyping involved the use of the HPV Sign ${ }^{\circledR}$ Genotyping (Qiagen, Hilden, Germany), or the INNO-LiPA ${ }^{\circledR}$ HPV Genotyping Extra assay (Innogenetics, Ghent, Belgium) or CLART ${ }^{\circledR}$ HPV2 PCR (Genomics, Madrid, Spain). The procedures mentioned above have been detailed in previous studies. ${ }^{21-24}$

\section{Statistical Methods}

Categorical variables were expressed as numbers and percentages. The Kolmogorov-Smirnov test was used to evaluate the distribution of continuous variables. Continuous variables not normally distributed were defined as median and interquartile ranges. Since our data expressed ordered categories, we used the Chi-Squared test for trend to measure the relationship between two classification factors (eg, periods and HPV genotypes or age). It is the right choice when a classification table has two columns and three or more rows (or two rows and three or more columns). ${ }^{25}$

The MedCalc ${ }^{\circledR}$ Statistical Software version 19.6 (MedCalc Software Ltd, Ostend, Belgium; https://www. medcalc.org; 2020) was used to perform statistical analyses. No formal statistical hypothesis testing is performed; so, we have not set a significance threshold. P-values should be considered continuous variables that report the probability of a difference being observed under the null hypothesis.

\section{Results}

The study period included 1325 consecutive unvaccinated women with CIN3 on cone histology. After excluding 851 cases, 474 women under 30 years were analyzed (Figure 1).

Patient characteristics are reported in Table 1. The median age was 26 years with an interquartile range of 25-28. Three hundred thirty-five participants $(70.7 \%)$ were positive for HPV 16/18; HPVs 31/33/35/52/58/67 were found in $16.2 \%$ of women; $4.4 \%$ of women were positive for HPV 39/45/59/68/70; 13.9\% of participants had HPV 31/33/45/52/58; possibly carcinogenic HPV and low-risk HPV were found in $2.3 \%$ and $3.0 \%$ of cases, respectively. Multiple infections amounted to 46.6\% (221 women). Time periods included 144 (2007-2010), 180 (2011-2014), and 150 (2015-2018) women, respectively. Different age groups had the following categories: $<25$ years (179 women) and 25-29 years (295 women). The majority of women were Italian (four hundred and sixteen, $87.8 \%$ ).

The distribution of high-risk HPV was the following: HPV-16 (58.9\%), HPV-18 (18.4\%), HPV-31 (11.2\%), HPV-52 (9.9\%) (Table 2). HPV 16/18 prevalence showed the following trend over time from 2007-2018: $77.8 \%$ vs $68.9 \%$ vs $66.0 \%$, respectively, (Ptrend $=0.027$ ) (Table 3). HPV 31/33/35/52/58/67 had the following trend over the years: $11.8 \%$ vs $17.8 \%$ vs $18.7 \%$, respectively, (Ptrend=0.112) (Table 3). Finally, there was a significant negative prevalence trend over the years for genotypes $26 / 30 / 53 / 67 / 70 / 73 / 82 / 85$ (4.9\% vs $1.1 \%$ vs $1.3 \%$, respectively, Ptrend $=0.046$ ) (Table 3 ). The agerelated trend of HPV genotypes assessed in the study was reported in Table 4. Over the years, there was a significant positive trend for high-risk HPV genotypes $31 / 33 / 45 / 52 / 58$ in women under $25(9.9 \%$ vs $17.0 \%$ vs $24.0 \%$, respectively, Ptrend $=0.048$ ) (Table 4).

Multiple HPV infections prevalence showed a significant negative trend over time from 2007-2018: 


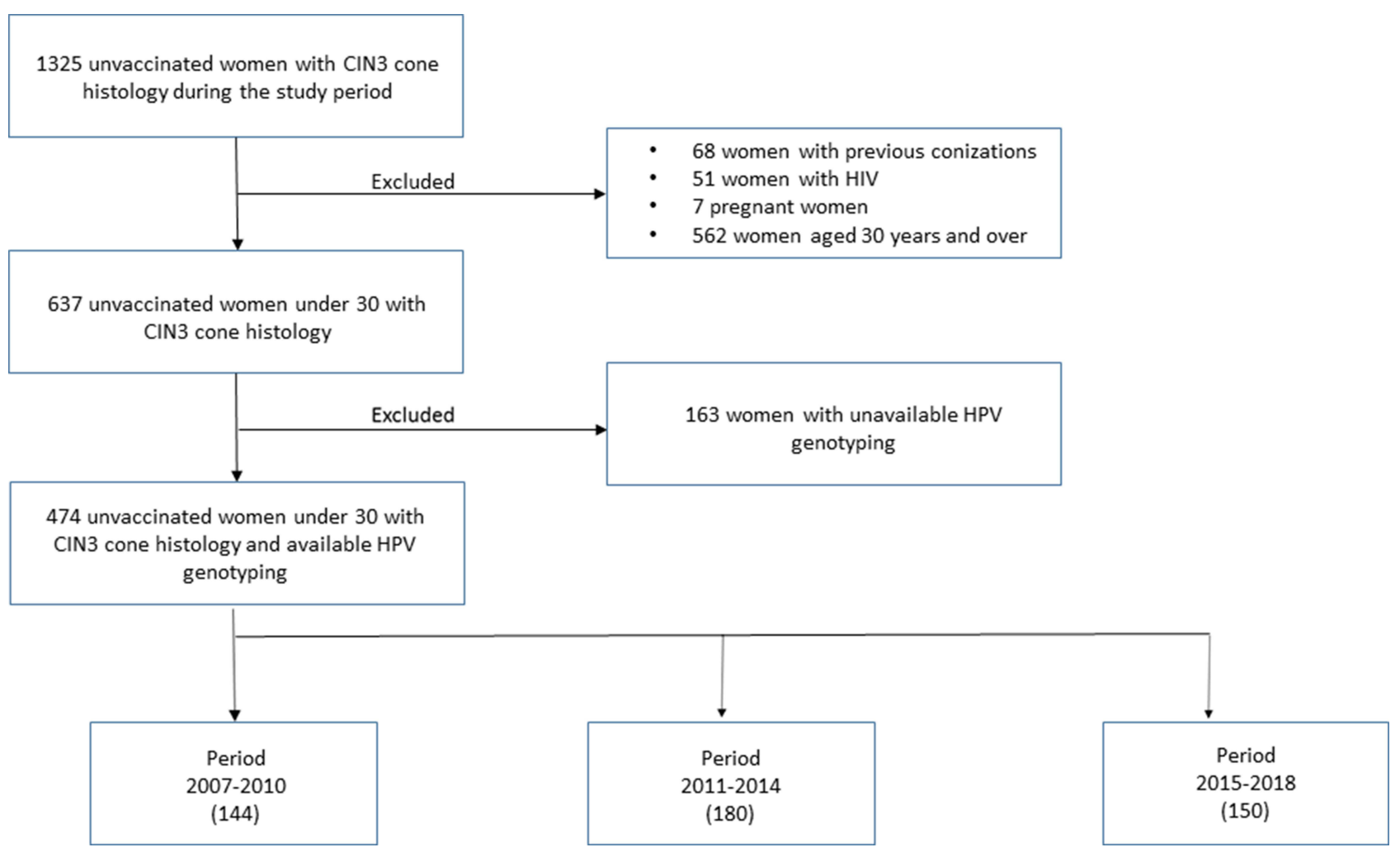

Figure I Study flow-chart.

$59.0 \%, 51.7 \%, 28.7 \%$, respectively $(\mathrm{p}<0.0001)$ (Figure 2$)$. The most significant reduction of multiple HPV infections over time was found in women aged 25-29: 60.5\%, $52.0 \%, 28.0 \%$, respectively ( $<<0.0001$ ) (Figure 2 ).

\section{Discussion}

The present study showed significant negative trends over time of HPV 16/18 infections in women with CIN3 after HPV vaccine introduction. There was a reduction of possibly carcinogenic HPV (genotypes 26/30/53/67/70/73/82/ $85)$. In women under 25 , there was a significant positive trend over time in high-risk HPV 31/33/45/52/58. Finally, the prevalence of multiple HPV infections decreased over time from 2007-2018.

Several authors have already demonstrated the efficacy of HPV vaccines. Previous studies showed a reduction in 4-valent-vaccine-type HPV among vaccinated women from $35.0 \%$ to $6.7 \%$, and unvaccinated women from $32.4 \%$ to $19.4 \% .{ }^{9,26}$ In a recent study evaluating the period from 2008-2014, a reduction in the proportion of CIN2+ lesions linked to HPV 16/18 was reported in vaccinated and unvaccinated women aged $18-39$ years. ${ }^{27}$ In contrast, a further study showed no trends in unvaccinated women aged 18-32 years with CIN3/AIS lesions linked to HPV
16/18 from 2011-2014. ${ }^{28}$ The latter study found the only negative trend of HPV 16/18 CIN3/AIS lesions in vaccinated women aged $18-25 .{ }^{28}$ These different findings may likely be due to various factors:

- Histological reference standards (CIN2+ versus CIN3/AIS);

- Different age courts (18-39 years versus 18-32 years);

- Different post-vaccination periods (2008-2014 versus 2011-2014).

In the present study, we included women with CIN3 diagnosis on cone specimens under 30 years. This age group may be considered the most representative for measuring an indirect impact of the vaccine in women of screening age. Our population included almost exclusively Italian women who showed a vaccination coverage of $61.7 \%$ for the first dose in the female population. ${ }^{14} \mathrm{We}$ reported a slight but significant reduction of HPV 16/18 and possibly carcinogenic HPV in women with high-grade cervical lesions on cone histology. This data support crossprotection of bivalent and quadrivalent vaccines against non-high-risk HPV genotypes. ${ }^{29}$ In women under 25 years, 
Table I Patient Characteristics

\begin{tabular}{|c|c|}
\hline Variables & Sample Size (474) n (\%) \\
\hline HPV I6/18 & $335(70.7)$ \\
\hline HPV-3I/33/35/52/58/67* & $77(16.2)$ \\
\hline HPV-39/45/59/68/70** & $21(4.4)$ \\
\hline HPV-3I/33/45/52/58*** & $66(13.9)$ \\
\hline HPV-26/30/53/67/70/73/82/85**** & II (2.3) \\
\hline HPV-6/II/40/42/43/44/54/55/6I***** & $14(3.0)$ \\
\hline Single HPV infections & $253(53.4)$ \\
\hline Multiple HPV infections & $221(46.6)$ \\
\hline \multicolumn{2}{|l|}{ Periods analyzed } \\
\hline $2007-2010$ & $144(30.4)$ \\
\hline $2011-2014$ & $180(38.0)$ \\
\hline $2015-2018$ & $150(31.6)$ \\
\hline \multicolumn{2}{|l|}{ Age-group } \\
\hline$<25$ years & $179(37.7)$ \\
\hline $25-29$ years & $295(62.3)$ \\
\hline \multicolumn{2}{|l|}{ Ethnicity } \\
\hline Italian & $416(87.8)$ \\
\hline Eastern European & $33(6.9)$ \\
\hline North European & $4(0.9)$ \\
\hline American & $9(1.9)$ \\
\hline African & $6(1.3)$ \\
\hline Asian & $6(1.3)$ \\
\hline
\end{tabular}

Notes: *Genetically related to HPVI6; **Genetically related to HPVI8; ***Highrisk-types included in the nonavalent vaccine; ****Possibly carcinogenic HPV; ******Low-risk HPV.

Abbreviations: HPV, Human Papillomavirus; I.R., interquartile range.

Table 2 Distribution of High-Risk HPV in Women Under 30 with CIN3

\begin{tabular}{|l|c|}
\hline HPV Genotypes & n (\%) \\
\hline HPV-16 & $279(58.9)$ \\
HPV-18 & $87(18.4)$ \\
HPV-3I & $53(11.2)$ \\
HPV-33 & $39(8.2)$ \\
HPV-35 & $8(1.7)$ \\
HPV-39 & $22(4.6)$ \\
HPV-45 & $8(1.7)$ \\
HPV-5I & $29(6.1)$ \\
HPV-52 & $47(9.9)$ \\
HPV-56 & $18(3.8)$ \\
HPV-58 & $26(5.5)$ \\
HPV-59 & $13(2.7)$ \\
HPV-66 & $20(4.2)$ \\
HPV-68 & $9(1.9)$ \\
\hline
\end{tabular}

Abbreviations: HPV, Human Papillomavirus; CIN, cervical intraepithelial neoplasia. we found a significant positive trend over time of high-risk HPVs included in the nonvalent vaccine. This data is likely affected by the recent introduction of the nonavalent vaccine. So these results will need to be reassessed in the coming years when this specific vaccine will probably achieve broader vaccination coverage.

We also showed a decreased prevalence over time of multiple HPV infections. This result may be due to the negative trend over time of the HPV-16 genotype. ${ }^{30}$ This reduced prevalence can have clinical implications. As demonstrated by previous authors, the reduction of multiple infections, including HPV 16 genotype, was associated with a lower risk of precancerous or invasive cervical lesions. $^{30-32}$ Furthermore, a decreased prevalence of multiple HPV subtypes was protective against new HPV infections. $^{31,32}$

Given the topic, these findings should be discussed on the possible impact on cervical screening. To date, cervical cancer prevention guidelines do not include vaccine status among variables assessing the risk of having/developing $\mathrm{CIN} 3$ as the best surrogate of CC. However, simulation studies showed scenarios with longer screening intervals in the post-vaccination era shifting the starting age to 30 years. ${ }^{15,33}$ Given the protective effect of HPV vaccines and the high sensitivity of the HPV test, it has been hypothesized that only two-lifetime screening tests may be sufficient in the near future. ${ }^{33}$ Nevertheless, this "one size fits all" screening policy, including vaccinated and unvaccinated women equally, could be envisaged if the minimum herd immunity threshold is reached. The present findings showed that we are still a long way from considering a "one size fits all" screening strategy. Currently, only a tailored screening protocol can be taken into account according to vaccine status. In this case, as reported by a recent Italian consensus conference, the Scientific Technical Committee invites the Italian Regions to link together the lists of vaccinated women and the lists of women invited or who have participated in the screening, in compliance with the legislation on personal data protection. ${ }^{15}$ Unvaccinated women should continue to follow the current cervical cancer screening recommendations. ${ }^{15}$

Another aspect being addressed is the oncogenic potential of cervical lesions according to the related HPV genotype. Indirect observations and prospective studies on HPV types in cancers showed that non-screening or nonvaccine type HPV have a lower cancerogenic potential. ${ }^{34}$ ${ }^{36}$ In other words, uncommon HPV-related CIN3 cervical lesions may have a lower chance of progression to cancer. In this regard, their progression from CIN1 to cancer may 
Table 3 Distribution of HPV Genotypes According to Different Periods in Women Under 30 with CIN3

\begin{tabular}{|l|c|c|c|c|}
\hline \multirow{2}{*}{ HPV Genotypes } & 2007-20 I0 n (\%) & 20I I-20I4 n (\%) & 20I5-20I8 n (\%) & P value \\
\cline { 2 - 5 } & Sample Size (144) & Sample Size (180) & Sample Size (150) \\
\hline HPV-I6/I8 & $112(77.8)$ & $124(68.9)$ & $99(66.0)$ & 0.027 \\
HPV-3I/33/35/52/58/67* & $17(11.8)$ & $32(17.8)$ & $28(18.7)$ & 0.112 \\
HPV-39/45/59/68/70** & $7(4.9)$ & $10(5.6)$ & $4(2.7)$ & 0.354 \\
HPV-3I/33/45/52/58*** & $14(9.7)$ & $28(15.6)$ & $24(16.0)$ & 0.122 \\
HPV-26/30/53/67/70/73/82/85**** & $7(4.9)$ & $2(1.1)$ & $2(1.3)$ & 0.046 \\
HPV-6/II/40/42/43/44/54/55/6I***** & $14(9.7)$ & $28(15.6)$ & $24(16.0)$ & 0.122 \\
\hline
\end{tabular}

Notes: *Genetically related to HPVI6; **Genetically related to HPVI8; ***High-risk-types included in the nonavalent vaccine; ****Possibly carcinogenic HPV; *****Low-risk HPV. Abbreviations: HPV, Human Papillomavirus; CIN, cervical intraepithelial neoplasia.

Table 4 Age-Related Trend of HPVs According to Different Periods in Women Under 30 with CIN3

\begin{tabular}{|c|c|c|c|c|c|}
\hline \multicolumn{2}{|l|}{ HPV Genotypes and Age-Groups } & \multirow{2}{*}{$\begin{array}{c}2007-2010 \text { n (\%) } \\
\text { Sample Size (144) }\end{array}$} & \multirow{2}{*}{$\begin{array}{c}20 \text { I I-20I } 4 \text { n (\%) } \\
\text { Sample Size (180) }\end{array}$} & \multirow{2}{*}{$\begin{array}{c}2015-2018 \text { n (\%) } \\
\text { Sample Size (150) }\end{array}$} & \multirow[t]{2}{*}{$P$ value } \\
\hline & & & & & \\
\hline \multirow[t]{2}{*}{ HPV-16/I8 } & Age $<25$ & $78(77.2)$ & 38 (7I.7) & $17(68.0)$ & 0.284 \\
\hline & Age 25-29 & $34(79.1)$ & $86(67.7)$ & $82(65.6)$ & 0.145 \\
\hline \multirow[t]{2}{*}{ HPV-3I/33/35/52/58/67* } & Age $<25$ & $12(11.9)$ & $10(18.9)$ & $5(20.0)$ & 0.197 \\
\hline & Age 25-29 & $5(1 \mathrm{l} .6)$ & $22(17.3)$ & $23(18.4)$ & 0.364 \\
\hline \multirow[t]{2}{*}{ HPV-39/45/59/68/70** } & Age $<25$ & $5(5.0)$ & I (I.9) & $0(0.0)$ & 0.159 \\
\hline & Age 25-29 & $2(4.7)$ & $9(7.1)$ & $4(3.2)$ & 0.412 \\
\hline \multirow[t]{2}{*}{ HPV-3I/33/45/52/58**** } & Age $<25$ & $10(9.9)$ & $9(\mid 7.0)$ & $6(24.0)$ & 0.048 \\
\hline & Age 25-29 & $4(9.3)$ & $19(15.0)$ & $18(14.4)$ & 0.532 \\
\hline \multirow[t]{2}{*}{ HPV-26/30/53/67/70/73/82/85**** } & Age $<25$ & $4(4.0)$ & $0(0.0)$ & $0(0.0)$ & 0.107 \\
\hline & Age $25-29$ & $3(7.0)$ & $2(1.6)$ & $2(1.6)$ & 0.108 \\
\hline \multirow[t]{2}{*}{ HPV-6/I I/40/42/43/44/54/55/6I****** } & Age $<25$ & $3(3.0)$ & $3(5.7)$ & I (4.0) & 0.604 \\
\hline & Age 25-29 & $0(0.0)$ & $6(4.7)$ & I (0.8) & 0.606 \\
\hline
\end{tabular}

Notes: *Genetically related to HPVI6; **Genetically related to HPVI8; ***High-risk- types included in the nonavalent vaccine; ****Possibly carcinogenic HPV; *****kow-risk HPV. Abbreviations: HPV, Human Papillomavirus; CIN, cervical intraepithelial neoplasia.

take longer to reach an appreciable proportion of preinvasive/invasive lesions. ${ }^{37}$ This slower progression may provide a greater chance of detecting these lesions at a preinvasive stage in a screening program. On the other hand, these high-grade lesions related to uncommon HPVs may likely have a greater regression chance. This could reduce excisional treatments among women of childbearing age, improving future obstetric outcomes. ${ }^{38,39}$ All these clinical implications underscore the importance of increasing HPV vaccination coverage.

The present study has the following limitations: a) its retrospective study design; b) the lack of vaccinated women in the sample analyzed (they would have allowed a complete evaluation of HPV infections over time); c) the time elapsed between HPV test and excisional treatment is not precisely known; d) study participants were not subjected to the same genotyping test. In the Centers participating in the study, the time interval between decision and surgical treatment usually does not go beyond four weeks. Finally, it is emphasized that the HPV Sign ${ }^{\circledR}$ Genotyping Test and INNO-LiPA ${ }^{\circledR}$ HPV Genotyping Extra showed an agreement rate of $85.1 \%{ }^{22}$

The study's strengths include the reliability of the histological standard based on cone histology and not cervical biopsy. And the better histopathological reproducibility of CIN3 than other high-grade lesions, such as CIN2. ${ }^{40}$

\section{Conclusions}

To conclude, in a real-world setting, including a fair number of young women with CIN3, the prevalence of 


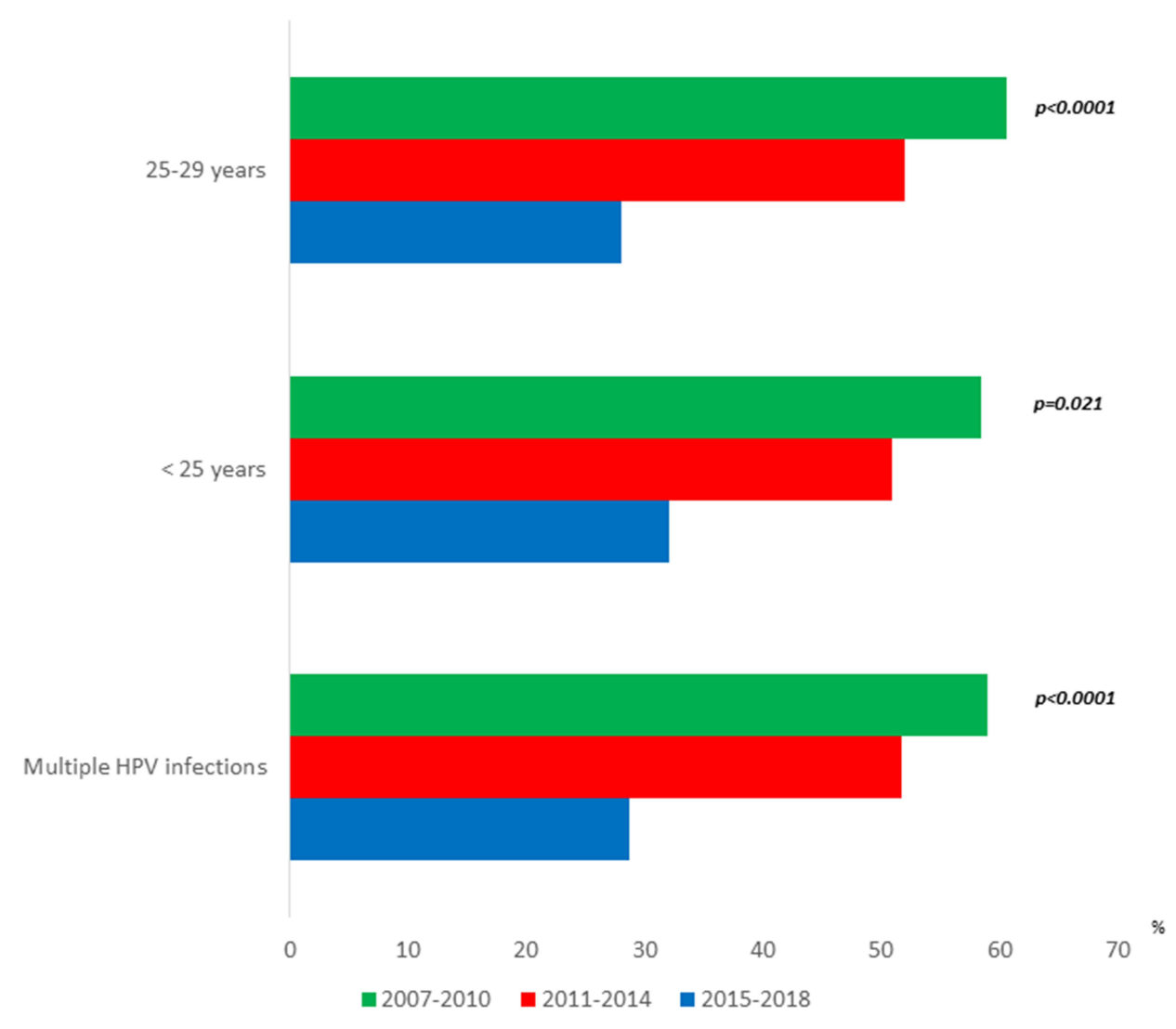

Figure 2 Age-related distribution of multiple HPV infections in different periods in $474 \mathrm{CIN} 3$.

precancerous cervical lesions mainly related to HPV 16/18 genotypes decreased over time from 2007-2018. These data highlight a herd effect of the HPV vaccine. However, about fifteen years after HPV vaccine introduction, we are still a long way from herd immunity in Italy based on these results. The increase in high-risk types $31 /$ $33 / 45 / 52 / 58$ will need to be reassessed in the coming years when the nonavalent vaccine impact will be more reliable.

\section{Data Sharing Statement}

The datasets are available from the corresponding author on reasonable request.

\section{Author Contributions}

All authors made a significant contribution to the work reported, whether that is in the conception, study design, execution, acquisition of data, analysis and interpretation, or in all these areas; took part in drafting, revising or critically reviewing the article; gave final approval of the version to be published; have agreed on the journal to which the article has been submitted; and agree to be accountable for all aspects of the work.

\section{Funding}

No funding was received for this study.

\section{Disclosure}

The authors declare no conflicts of interest.

\section{References}

1. Serrano B, Alemany L, Tous S, et al. Potential impact of a nine-valent vaccine in human papillomavirus related cervical disease. Infect Agent Cancer. 2012;7(1):38. doi:10.1186/17509378-7-38

2. Liverani CA, Di Giuseppe J, Giannella L, Delli Carpini G, Ciavattini A. Cervical cancer screening guidelines in the postvaccination era: review of the literature. J Oncol. 2020;2020:8887672. doi:10.1155/2020/8887672

3. Arbyn M, Xu L. Efficacy and safety of prophylactic HPV vaccines. A cochrane review of randomized trials. Expert Rev Vaccines. 2018;17(12):1085-1091. doi:10.1080/14760584.20 18.1548282

4. Ciavattini A, Giannella L, De Vincenzo R, et al. HPV vaccination: the position paper of the Italian society of colposcopy and cervico-vaginal pathology (SICPCV). Vaccines (Basel). 2020;8(3):E354. doi:10.3390/ vaccines 8030354

5. Centers for Disease Control and Prevention. HPV vaccine information for clinicians-fact sheet. Available from: https://www.cdc.gov/std/ HPV. Accessed September 8, 2021. 
6. Paz-Zulueta M, Álvarez-paredes L, Rodríguez Díaz JC, et al. Prevalence of high-risk HPV genotypes, categorised by their quadrivalent and nine-valent HPV vaccination coverage, and the genotype association with high-grade lesions. BMC Cancer. 2018;18(1):112. doi:10.1186/s12885-018-4033-2

7. Food and Drug Administration. FDA approves gardasil 9 for prevention of certain cancers caused by five additional types of HPV. Available From: https://www.esmo.org/oncology-news/archive/fdaapproves-gardasil-9-for-prevention-of-certain-cancers-caused-byfive-additional-types-of-hpv. Accessed September 8, 2021.

8. Joura EA, Ault KA, Bosch FX, et al. Attribution of 12 high-risk human papillomavirus genotypes to infection and cervical disease. Cancer Epidemiol Biomarkers Prev. 2014;23(10):1997-2008. doi:10.1158/1055-9965.epi-14-0410

9. Spinner C, Ding L, Bernstein DI, et al. Human papillomavirus vaccine effectiveness and herd protection in young women. Pediatrics. 2019;143(2):e20181902. doi:10.1542/peds.2018-1902

10. Safaeian M, Rodriguez AC. Invited commentary: multiple human papillomavirus infections and type replacement-anticipating the future after human papillomavirus vaccination. Am J Epidemiol. 2014;180(11):1076-1081. doi:10.1093/aje/kwu265

11. Cornall AM, Phillips S, Cummins E, Garland SM, Tabrizi SN. In vitro assessment of the effect of vaccine-targeted human papillomavirus (HPV) depletion on detection of non-vaccine HPV types: implications for post-vaccine surveillance studies. $J$ Virol Methods. 2015;214:10-14. doi:10.1016/j.jviromet.2014.12.007

12. Stanley M, Lowy DR, Frazer I. Chapter 12: prophylactic HPV vaccines: underlying mechanisms. Vaccine. 2006;24 Suppl 3: S3/106-13.

13. Istituto Superiore di Sanità. Available from: https://www.epicentro. iss.it/hpv/epidemiologia-cervicocarcinoma-italia. Accessed September 8, 2021.

14. Istituto Superiore di Sanità. Available from: https://www.epicentro. iss.it/vaccini/dati_Ita\#hpv. Accessed September 8, 2021.

15. Giorgi Rossi P, Carozzi F, Federici A, Ronco G, Zappa M, Franceschi S. Italian screening in HPV vaccinated girls consensus conference group. Cervical cancer screening in women vaccinated against human papillomavirus infection: recommendations from a consensus conference. Prev Med. 2017;98:21-30. doi:10.1016/j. ypmed.2016.11.020

16. Perkins RB, Guido RS, Castle PE, et al. 2019 ASCCP risk-based management consensus guidelines for abnormal cervical cancer screening tests and cancer precursors. J Low Genit Tract Dis. 2020;24(2):102-131. doi:10.1097/LGT.0000000000000525

17. Gazzetta Ufficiale. Available from: https://www.gazzettaufficiale.it/ eli/gu/2012/03/26/72/sg/pdf. Accessed September 8, 2021.

18. Rositch AF, Soeters HM, Offutt-Powell TN, Wheeler BS, Taylor SM, Smith JS. The incidence of human papillomavirus infection following treatment for cervical neoplasia: a systematic review. Gynecol Oncol. 2014;132:767-779. doi:10.1016/j.ygyno.2013.12.040

19. IARC Working Group on the Evaluation of Carcinogenic Risks to Humans. IARC monographs on the evaluation of carcinogenic risks to humans. Ingested nitrate and nitrite, and cyanobacterial peptide toxins. IARC Monogr Eval Carcinog Risks Hum. 2012;100(Pt B): $1-441$.

20. Perez S, Iñarrea A, Pérez-Tanoira R, et al. Fraction of high-grade cervical intraepithelial lesions attributable to genotypes targeted by a nonavalent HPV vaccine in Galicia, Spain. Virol J. 2017;14:214. doi:10.1186/s12985-017-0879-1

21. Giannella L, Delli Carpini G, Di Giuseppe J, Prandi S, Tsiroglou D, Ciavattini A. Age-related changes in the fraction of cervical intraepithelial neoplasia grade 3 related to HPV genotypes included in the nonavalent vaccine. J Oncol. 2019;2019:7137891. doi:10.1155/2019/ 7137891
22. Barbieri D, Nocera M, Gallinella G, et al. Comparison of HPV sign genotyping test with INNO-LiPA HPV genotyping extra assay on histologic and cytologic cervical specimens. Diagn Microbiol Infect Dis. 2012;74(1):43-48. doi:10.1016/j.diagmicrobio.2012.05.014

23. Pichon M, Lebail-Carval K, Billaud G, Lina B, Gaucherand P, Mekki Y. Decontamination of intravaginal probes infected by human papillomavirus (HPV) using UV-C decontamination system. J Clin Med. 2019;8:1776. doi:10.3390/jcm8111776

24. Spinillo A, Dominoni M, Boschi AC, et al. Clinical significance of the interaction between human papillomavirus (HPV) type 16 and other high-risk human papillomaviruses in women with cervical intraepithelial neoplasia (CIN) and invasive cervical cancer. J Oncol. 2020;26:6508180.

25. Armitage P. Tests for linear trends in proportions and frequencies. Biometrics. 1955;11(3):375-386. doi:10.2307/3001775

26. Covert C, Ding L, Brown D, Franco EL, Bernstein DI, Kahn JA. Evidence for cross-protection but not type-replacement over the 11 years after human papillomavirus vaccine introduction. Hum Vaccin Immunother. 2019;15(7-8):1962-1969. doi:10.1080/21645515.2018.1564438

27. McClung NM, Gargano JW, Bennett NM, et al. Trends in human papillomavirus vaccine types 16 and 18 in cervical precancers, 20082014. Cancer Epidemiol Biomarkers Prev. 2019;28(3):602-609. doi:10.1158/1055-9965.EPI-18-0885

28. Cornall AM, Saville M, Pyman J; VACCINE Study Group. HPV16/ 18 prevalence in high-grade cervical lesions in an Australian population offered catch-up HPV vaccination. Vaccine. 2020;38 (40):6304-6311. doi:10.1016/j.vaccine.2020.07.037

29. Reich O, Regauer S, Kashofer K. Possibly carcinogenic HPV subtypes are a cause of HSIL and negative clinical HPV tests A European prospective single center study. Gynecol Oncol. 2020;158(1):112-116. doi:10.1016/j.ygyno.2020.04.685

30. Bruno MT, Scalia G, Cassaro N, Boemi S. Multiple HPV 16 infection with two strains: a possible marker of neoplastic progression. $B M C$ Cancer. 2020;20(1):444. doi:10.1186/s12885-020-06946-7

31. Lee SA, Kang D, Seo SS, et al. Multiple HPV infection in cervical cancer screened by HPVDNAChip. Cancer Lett. 2003;198:187-192. doi:10.1016/s0304-3835(03)00312-4

32. Goodman MT, McDuffie K, Hernandez BY, et al. The influence of multiple human papillomavirus types on the risk of genotype-concordant incident infections of the anus and cervix: the Hawaii HPV cohort study. J Infect Dis. 2011;203:335-340. doi:10.1093/infdis/jiq058

33. Landy R, Windridge P, Gillman MS, Sasieni PD. What cervical screening is appropriate for women who have been vaccinated against high risk HPV? A simulation study. Int $J$ Cancer. 2018;142:709-718. doi:10.1002/ijc.31094

34. Guan P, Howell-Jones R, Li N, et al. Human papillomavirus types in 115,789 HPV-positive women: a meta-analysis from cervical infection to cancer. Int J Cancer. 2012;131(10):2349-2359. doi:10.1002/ ijc. 27485

35. Castle PE, Kinney WK, Xue X, et al. Effect of several negative rounds of human papillomavirus and cytology co-testing on safety against cervical cancer: an observational cohort study. Ann Intern Med. 2018;168(1):20-29. doi:10.7326/M17-1609

36. Carozzi FM, Tornesello ML, Burroni E, et al.; HPV Prevalence Italian Working Group. Prevalence of human papillomavirus types in high-grade cervical intraepithelial neoplasia and cancer in Italy. Cancer Epidemiol Biomarkers Prev. 2010;19(9):2389-2400. doi:10.1158/1055-9965.EPI-10-0131

37. Giannella L, Giorgi Rossi P, Delli Carpini G, et al. Age-related distribution of uncommon HPV genotypes in cervical intraepithelial neoplasia grade 3. Gynecol Oncol. 2021;161(3):741-747. doi:10.1016/j.ygyno.2021.03.025 
38. Giannella L, Beraldi R, Giulini S, Cerami LB, Mfuta K, Facchinetti F. Nitric oxide metabolite levels and assessment of cervical length in the prediction of preterm delivery among women undergoing symptomatic preterm labor. Int J Gynaecol Obstet. 2012;116 (3):223-227. doi:10.1016/j.ijgo.2011.10.020

39. Kyrgiou M, Athanasiou A, Kalliala IEJ, et al. Obstetric outcomes after conservative treatment for cervical intraepithelial lesions and early invasive disease. Cochrane Database Syst Rev. 2017;11: CD012847.
40. Dalla PP, Giorgi RP, Collina G, Ronco G; NTCC Pathology Group. The reproducibility of CIN diagnoses among different pathologists: data from histology reviews from a multicenter randomized study. Am J Clin Pathol. 2009;132(1):125-132. doi:10.1309/AJCPBRK7D1YIUWFP

\section{Publish your work in this journal}

Infection and Drug Resistance is an international, peer-reviewed openaccess journal that focuses on the optimal treatment of infection (bacterial, fungal and viral) and the development and institution of preventive strategies to minimize the development and spread of resistance. The journal is specifically concerned with the epidemiology of

Submit your manuscript here: https://www.dovepress.com/infection-and-drug-resistance-journa antibiotic resistance and the mechanisms of resistance development and diffusion in both hospitals and the community. The manuscript management system is completely online and includes a very quick and fair peerreview system, which is all easy to use. Visit http://www.dovepress.com/ testimonials.php to read real quotes from published authors. 